\title{
Îndice Hemodinâmico Agudo Prediz Mortalidade Intra-Hospitalar de Pacientes com Insuficiência Cardíaca Aguda Descompensada
}

\author{
Acute Hemodynamic Index Predicts In-Hospital Mortality in Acute Decompensated Heart Failure \\ Renata R. T. Castro, ${ }^{1,2,3}{ }^{10}$ Luka Lechnewski, ${ }^{4}$ Alan Homero, ${ }^{4}$ Denilson Campos de Albuquerque, ${ }^{5}$ Luis Eduardo \\ Rohde, ${ }^{6}$ Dirceu Almeida, ${ }^{70}$ João David, ${ }^{8}$ Salvador Rassi, ${ }^{9}$ Fernando Bacal, ${ }^{10}$ Edimar Bocchi, ${ }^{10}$ Lidia Moura ${ }^{4}$ \\ Brigham and Womens Hospital - Medicine, ${ }^{1}$ Boston - EUA \\ Hospital Naval Marcilio Dias, ${ }^{2}$ Rio de Janeiro, $R J$ - Brasil \\ Faculdade de Medicina, Universidade Iguaçu, ${ }^{3}$ Nova Iguaçu, RJ - Brasil \\ Pontifícia Universidade Católica do Paraná, ${ }^{4}$ Curitiba, $P R$ - Brasil \\ Universidade Estadual do Rio de Janeiro, ${ }^{5}$ Rio de Janeiro, RJ - Brasil \\ Universidade Federal do Rio Grande do Sul, ${ }^{6}$ Porto Alegre, RS - Brasil \\ Universidade Federal de São Paulo, ${ }^{7}$ São Paulo, SP - Brasil \\ Hospital de Messejana, ${ }^{8}$ Fortaleza, CE - Brasil \\ Universidade Federal de Goiás, ${ }^{9}$ Goiânia, GO - Brasil \\ Universidade de São Paulo Instituto do Coração, ${ }^{10}$ São Paulo, SP - Brasil
}

\section{Resumo}

Fundamento: O exame físico permite a avaliação prognóstica de pacientes com insuficiência cardíaca (IC) descompensada, porém não é suficientemente confiável e depende da experiência clínica do profissional. Considerando as respostas hemodinâmicas a situações do tipo "luta ou fuga" tais como a admissão no serviço de emergência, foi proposto o índice hemodinâmico agudo (IHA), calculado a partir da frequência cardíaca e pressão de pulso.

Objetivo: avaliar a capacidade prognóstica intra-hospitalar do IHA na IC descompensada.

Métodos: estudo prospectivo, multicêntrico e observacional baseado no registro BREATHE, incluindo dados de hospitais públicos e privados no Brasil. Foram utilizadas análises ROC (Receiver Operating Characteristic), de estatística c e de regressão multivariada, assim como o critério de informação de Akaike, para testar a capacidade prognóstica do IHA. $O$ valor-p $<0,05$ foi considerado estatisticamente significativo.

Resultados: Foram analisados dados de 463 pacientes com IC com fração de ejeção reduzida a partir do registro BREATHE. A mortalidade intra-hospitalar foi de $9 \%$. A mediana do IHA foi considerada o valor de corte $(4 \mathrm{mmHg} \cdot \mathrm{bpm})$. Um baixo IHA ( $\leq 4 \mathrm{mmHg} \cdot \mathrm{bpm}$ ) foi encontrado em $80 \%$ dos pacientes falecidos. $O$ risco de mortalidade intrahospitalar em pacientes com baixo IHA foi 2,5 vezes maior que aquele para pacientes com IHA $>4 \mathrm{mmHg} \cdot \mathrm{bpm}$. $\mathrm{O}$ IHA foi capaz de predizer independentemente a mortalidade intra-hospitalar na IC aguda descompensada [sensibilidade: 0,786; especificidade: 0,429; AUC (área sob a curva): 0,607 (0,540-0,674), $p=0,010$ ] mesmo depois dos ajustes para comorbidades e uso de medicamentos [razão de chances (RC): 0,061 (0,007-0,114), p = 0,025].

Conclusões: O IHA é capaz de predizer independentemente a mortalidade intra-hospitalar na IC aguda descompensada. Esse índice simples e realizado à beira do leito pode se mostrar útil em serviços de emergência. (Arq Bras Cardiol. 2021; 116(1):77-86)

Palavras-chave: Insuficiência Cardíaca; Frequência Cardíaca; Pressão Arterial; Prognóstico; Mortalidade.

\footnotetext{
Abstract

Background: The physical examination enables prognostic evaluation of patients with decompensated heart failure (HF), but lacks reliability and relies on the professional's clinical experience. Considering hemodynamic responses to "fight or flight" situations, such as the moment of admission to the emergency room, we proposed the calculation of the acute hemodynamic index (AHI) from values of heart rate and pulse pressure.
}

Objective: To evaluate the in-hospital prognostic ability of AHI in decompensated HF.

Correspondência: Renata R. T. Castro •

Universidade Iguaçu - Rua Abílio Augusto Távora, 2134. CEP 26260-045 Nova Iguaçu, RJ - Brasil

E-mail: castrorrt@gmail.com

Artigo recebido em 29/09/2019, revisado em 15/02/2020, aceito em 16/03/2020

DOI: https://doi.org/10.36660/abc.20190439 
Methods: A prospective, multicenter, registry-based observational study including data from the BREATHE registry, with information from public and private hospitals in Brazil. The prognostic ability of the AHI was tested by receiver-operating characteristic (ROC) analyses, C-statistics, Akaike's information criteria, and multivariate regression analyses. p-values $<0.05$ were considered statistically significant.

Results: We analyzed data from 463 patients with heart failure with low ejection fraction. In-hospital mortality was $9 \%$. The median AHI value was used as cut-off (4 mmHg.bpm). A low AHI ( $\leq 4 \mathrm{mmHg} \cdot \mathrm{bpm})$ was found in $80 \%$ of deceased patients. The risk of inhospital mortality in patients with low AHI was 2.5 times that in patients with AHI $>4 \mathrm{mmHg} \cdot \mathrm{bpm}$. AHI independently predicted in-hospital mortality in acute decompensated HF (sensitivity: 0.786; specificity: 0.429; AUC: 0.607 [0.540-0.674]; $p=0.010$ ) even after adjusting for comorbidities and medication use [OR: $0.061(0.007-0.114) ; p=0.025)$.

Conclusions: The AHI independently predicts in-hospital mortality in acute decompensated HF. This simple bed-side index could be useful in an emergency setting. (Arq Bras Cardiol. 2021; 116(1):77-86)

Keywords: Heart Failure; Heart Rate; Blood Pressure; Prognosis; Mortality.

Full texts in English - http://www.arquivosonline.com.br

\section{Introdução}

A insuficiência cardíaca (IC) é uma das principais razões de admissões hospitalares de emergência no mundo ocidental. ${ }^{1}$ Apesar de estudos terem mostrado que o tratamento com um especialista em IC pode fornecer melhores resultados, a maioria dos casos de descompensação aguda da IC são originalmente avaliados e manejados por médicos emergencistas ${ }^{2,3} \mathrm{em}$ instituições com diferentes disponibilidades de recursos.

Mesmo com o avanço da tecnologia e dos dispositivos médicos, o exame físico continua sendo o pilar principal da avaliação de pacientes com IC. ${ }^{4,5}$ Médicos avaliam a congestão e perfusão a partir do histórico do paciente e do exame físico, determinando perfis hemodinâmicos que podem guiar a terapêutica e fornecer informações prognósticas numa situação de IC aguda. ${ }^{6}$ Apesar da praticidade, a avaliação da perfusão feita pelo médico não é suficientemente confiável $^{7}$ e depende da experiência do profissional, ${ }^{8,9}$ posto que fornece informações subjetivas. ${ }^{10}$ Logo, parâmetros prognósticos objetivos que podem ser facilmente obtidos em um atendimento de emergência seriam úteis no manejo da IC aguda.

A pressão arterial e a frequência cardíaca são parâmetros que podem ser facilmente obtidos por qualquer profissional de saúde, com boa reprodutibilidade e acurácia. ${ }^{11,12} \mathrm{~A}$ pressão arterial sistólica é um preditor independente de desfechos intra-hospitalares e pós-alta na IC aguda. ${ }^{13,14}$ Além disso, baixos valores de pressão arterial e pressão de pulso proporcional são indicadores de baixa perfusão. , $, 6,9^{2}$

A relação entre frequência cardíaca em repouso na admissão e o prognóstico de pacientes com IC não é completamente direta. Na realidade, a literatura apresenta resultados controversos, indicando que uma frequência cardíaca alta no momento da admissão pode estar relacionada a um prognóstico melhor ou pior. ${ }^{15-17}$ Apesar de baixas frequências cardíacas em repouso indicarem um menor risco em pacientes com IC crônica estável com fração de ejeção reduzida, ${ }^{18,19}$ é inegável que a capacidade de aumentar a frequência cardíaca numa reação do tipo "luta ou fuga" também confere um bom prognóstico, ${ }^{20,21}$ independentemente do uso de betabloqueadores.

A admissão aguda no serviço de emergência é uma situação de estresse onde se esperam respostas autonômicas que preparam o corpo para uma situação de luta ou fuga. ${ }^{22}$
Nesse contexto, aumentos na pressão de pulso e frequência cardíaca são esperados, aumentando a perfusão nos músculos esqueléticos e órgãos vitais.

Com base nas respostas hemodinâmicas fisiológicas inerentes a situações de luta ou fuga, propõe-se o cálculo do índice hemodinâmico agudo (IHA) a partir da frequência cardíaca e pressão de pulso. Nossa principal hipótese é que o IHA poderia representar um parâmetro prognóstico objetivo e intra-hospitalar a ser usado na descompensação aguda de pacientes com IC com fração de ejeção reduzida (ICFER). Portanto, o objetivo deste estudo foi avaliar a capacidade prognóstica intra-hospitalar do IHA na ICFER aguda descompensada.

\section{Métodos}

Esta análise foi baseada no I Registro Brasileiro de Insuficiência Cardíaca (BREATHE), ${ }^{23,24}$ um estudo observacional transversal tipo registro da IC aguda com seguimento longitudinal que ocorreu de fevereiro de 2011 a dezembro de 2012. Para inclusão no registro, pacientes deveriam possuir mais de 18 anos e ter sido admitidos com IC descompensada; os pacientes não poderiam ter sido submetidos a cirurgia de revascularização miocárdica ou intervenção coronária percutânea no mês anterior, nem ter sido admitidos com um diagnóstico de sepse. Os critérios de Boston foram utilizados para a confirmação da $\mathrm{IC} \cdot{ }^{25} \mathrm{~A}$ participação no registro não requeria nenhum regime de tratamento especial. Os métodos detalhados, assim como os critérios de inclusão e exclusão, estão disponíveis na literatura. ${ }^{24}$ As informações sobre cada paciente foram incluídas num formulário de registro individual, disponível online.

Este estudo incluiu a análise da admissão hospitalar e do seguimento dos pacientes até a alta médica, morte ou transferência para outro hospital (o que foi verificado primeiro) dos pacientes com ICFER aguda descompensada. O desfecho primário do estudo foi a mortalidade intra-hospitalar.

Todos os pacientes do registro com evidência de fração de ejeção do ventrículo esquerdo menor que $40 \%$ foram incluídos nesta análise, exceto aqueles com informações faltantes (frequência cardíaca na admissão, pressão arterial, fração de ejeção ou interrupção do seguimento devido à 
transferência). Indivíduos com ritmo cardíaco controlado por marca-passo também foram excluídos, visto que não era esperado que suas frequências cardíacas possuíssem um controle autonômico (Figura 1).

\section{Variáveis derivadas}

Frequência cardíaca e pressão arterial sistólica e diastólica na admissão foram obtidas a partir da base de dados do registro e utilizadas no cálculo das variáveis derivadas da seguinte maneira: pressão de pulso = pressão arterial sistólica - pressão arterial diastólica; pressão de pulso proporcional = pressão de pulso / pressão arterial sistólica; $\mathrm{IHA}=$ (Pressão de pulso $\mathrm{x}$ frequência cardíaca) / 1000

\section{Aspectos éticos}

Este estudo está de acordo com os princípios da Declaração de Helsinque. O estudo foi aprovado pelo Hospital do Coração, São Paulo (registro 144/2011) e pelo comitê de ética em pesquisa de cada instituição participante. Todos os pacientes assinaram um termo de consentimento esclarecido antes do recrutamento.

\section{Análise estatística}

Inicialmente, utilizado o teste de Shapiro Wilk para verificar a normalidade da distribuição dos dados, validando o uso da estatística paramétrica. Variáveis contínuas foram descritas como médias e desvios-padrão, e variáveis categóricas foram descritas como proporções. Dados clínicos

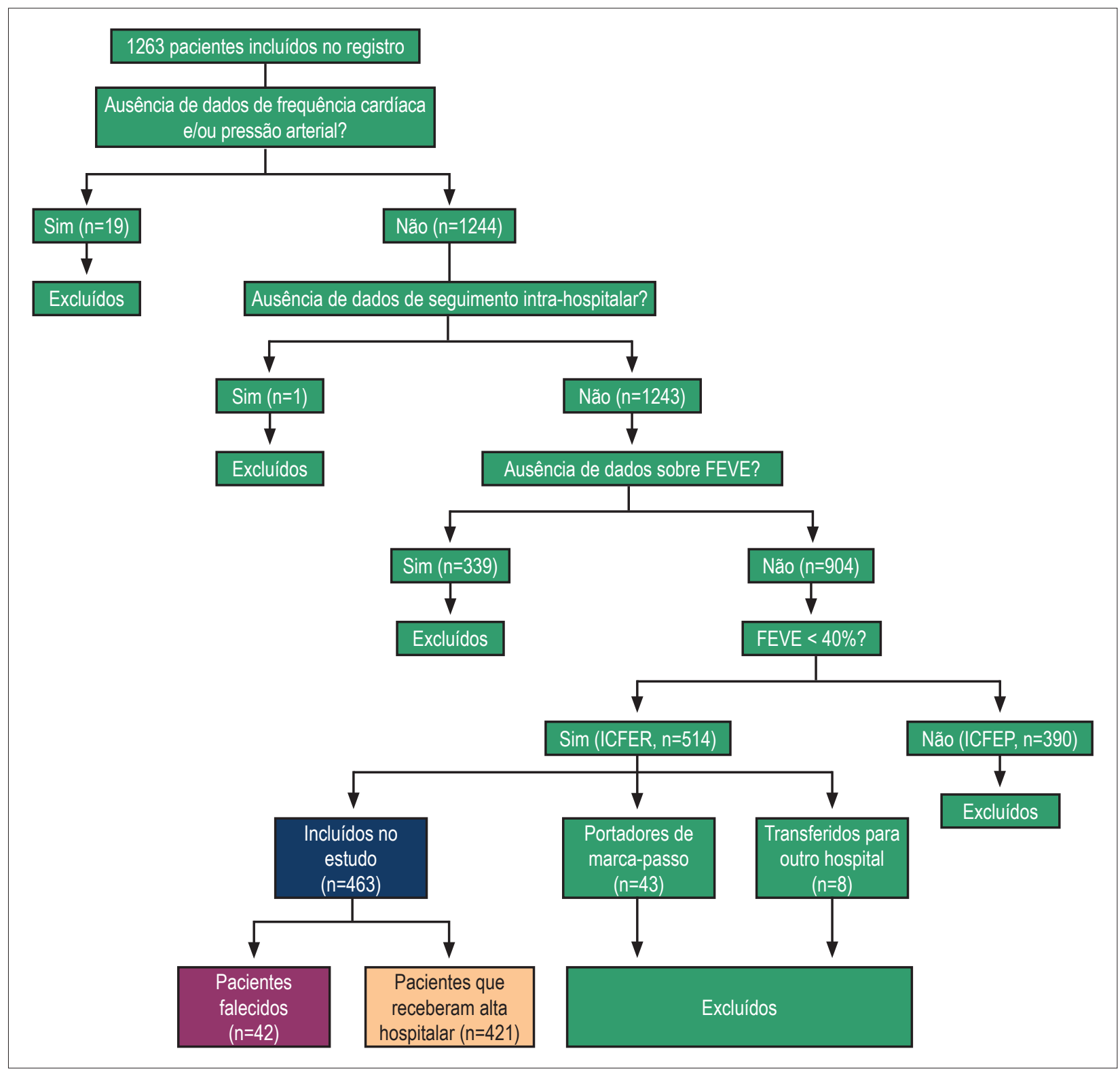

Figura 1 - Diagrama de seleção de pacientes para o registro. FEVE: fração de ejeção do ventrículo esquerdo; ICFER: insuficiência cardiaca com fração de ejeção reduzida; ICFEP: insuficiência cardíaca com fração de ejeção preservada. 
e demográficos dos pacientes que vieram a óbito durante a hospitalização (falecidos) e daqueles que receberam alta médica (vivos) foram comparados por um teste t de Student não-pareado ou qui-quadrado. Um valor-p bicaudal $<0,05$ foi considerado estatisticamente significativo.

Depois de verificar a distribuição normal, os percentis 25ํㅡ, $50^{\circ}$ e $75^{\circ}$ de frequência cardíaca e pressão arterial sistólica e diastólica foram utilizados na construção de curvas ROC (Receiver Operating Characteristic) usando a mortalidade intra-hospitalar como desfecho principal. O valor de corte do IHA foi definido pelo $50^{\circ}$ percentil de sua distribuição. Sensibilidade, especificidade, e área sob a curva ROC (AUC) foram estabelecidas para cada valor de corte. Estatística c foi usada para comparar a capacidade prognóstica dos valores de corte de frequência cardíaca e pressão arterial com os valores de corte de IHA.

A análise de regressão foi realizada após a verificação de relação linear, normalidade multivariada, homoscedasticidade e da ausência de multicolinearidade e autocorrelação. Foram realizadas análises de regressão linear múltipla para testar a capacidade prognóstica independente de cada um dos valores de corte significativos de frequência cardíaca, pressão arterial e IHA. Esta análise também incluiu variáveis com significância estatística de acordo com testes t não-pareado e quiquadrado. Visto que nem todos os pacientes apresentavam dados laboratoriais, estes não foram incluídos na análise de regressão. O critério de informação de Akaike (AIC) ${ }^{26}$ foi usado para comparar os modelos de regressão múltipla.

O software STATA versão 14.2 (StataCorp, Texas, EUA) foi utilizado em todas as análises estatísticas e gráficos.

\section{Resultados}

O registro BREATHE incluiu 463 pacientes com ICFER admitidos em serviços de emergência no Brasil (Tabela 1), com um índice de mortalidade intra-hospitalar de 9\%. A principal causa de descompensação foi má aderência ao tratamento medicamentoso (em 37\% dos pacientes que receberam alta e $31 \%$ daqueles que faleceram, $p=0,75)$. Outras causas importantes de descompensação foram infecções (em 21\% dos pacientes que receberam alta e $24 \%$ daqueles que faleceram, $p=0,17)$ e ingestão excessiva de sal ou fluidos (em $11 \%$ dos pacientes que receberam alta e $12 \%$ daqueles que faleceram, $p=0,90)$.

Os pacientes falecidos apresentavam mais comorbidades, assim como frequência cardíaca e pressão arterial sistólica e diastólica mais altas que aqueles que sobreviveram. Considerando o 50o percentil do IHA como valor de corte (4 $\mathrm{mmHg} \cdot \mathrm{bpm})$, quase $80 \%$ dos pacientes falecidos possuíam um baixo IHA.

Visto que o cálculo do IHA inclui valores de frequência cardíaca e pressão arterial, foram comparadas as AUC de $\mathrm{IHA} \leq 4 \mathrm{mmHg} \cdot \mathrm{bpm}$ e de diferentes valores de corte de frequência cardíaca e pressão arterial sistólica e diastólica (Tabela 2). IHA $\leq 4 \mathrm{mmHg} \cdot \mathrm{bpm}$ foi um melhor preditor de mortalidade intra-hospitalar que frequência cardíaca $\leq 88$ bpm, mas teve desempenho semelhante a outros valores de corte de pressão arterial. Quando estes fatores prognósticos hemodinâmicos foram incluídos na análise multivariada, apenas o IHA manteve capacidade prognóstica independente (Tabela 3). O modelo de regressão que incluiu a etiologia de doença de Chagas, comorbidades, uso de medicamentos e IHA mostrou uma melhor capacidade prognóstica de mortalidade intra-hospitalar que os outros modelos propostos (Modelo 0: sem IHA; Modelos 1-4: com parâmetros hemodinâmicos adicionados ao Modelo 0). Doença renal crônica, histórico de câncer ou acidente vascular cerebral permaneceram como preditores independentes de mortalidade intra-hospitalar em todos os modelos propostos. IHA $<4 \mathrm{mmHg} \cdot \mathrm{bpm}$ esteve independentemente relacionado à mortalidade intra-hospitalar neste registro mesmo após o ajuste para a etiologia da IC, comorbidades e uso de medicamentos (Figura 2). Pacientes admitidos com baixos IHAs tinham probabilidade de óbito de $12,1 \%$, um valor $250 \%$ maior que pacientes admitidos com IHAs maiores que $4 \mathrm{mmHg} \cdot \mathrm{bpm}$ $(4,8 \%, p=0,008$, Figura 3). Como trata-se de um estudo tipo registro, não houve intervenções no tratamento recebido pelos pacientes. Medicamentos inotrópicos foram utilizados em $11 \%$ dos pacientes que receberam alta e em $28 \%$ dos que vieram a óbito $(p<0,001)$.

\section{Discussão}

Este estudo apresentou o IHA e demonstrou que este é um preditor independente de mortalidade intra-hospitalar em pacientes com ICFER aguda descompensada. A mortalidade intra-hospitalar de pacientes com IC aguda descompensada é elevada, como demonstrado pelo registro BREATHE e por estudos realizados em outros países. ${ }^{27}$ Diferentes razões contribuem para uma alta mortalidade a curto prazo de pacientes com IC aguda descompensada, como idade, comorbidades e tempo entre início dos sintomas e admissão hospitalar. ${ }^{27}$ Visto que o manejo de pacientes com IC aguda pode incluir procedimentos invasivos e de alto custo, como o suporte circulatório mecânico, a validação de índices prognósticos que possam auxiliar em decisões terapêuticas é de extrema importância. ${ }^{28}$

A IC aguda descompensada pode ser manejada por especialistas em IC, cardiologistas, intensivistas, médicos emergencistas ou clínicos; em serviços de emergência, enfermarias ou unidades de tratamento intensivo. ${ }^{2} \mathrm{O}$ treinamento destes profissionais e a disponibilidade de recursos nestes ambientes pode variar substancialmente. Quando somados à diversidade de casos de pacientes, estes aspectos tornam difícil a criação de escores prognósticos que possam ser utilizados amplamente. Por exemplo, apesar do recente interesse no uso de biomarcadores, ${ }^{29}$ a avaliação destes pode não estar disponível em estabelecimentos de saúde afastados dos grandes centros ou com poucos recursos. Nohria et al. ${ }^{6}$ apresentaram uma abordagem clínica prática para classificar os pacientes em perfis hemodinâmicos, permitindo a predição do prognóstico e guiando o tratamento da IC aguda. Esta abordagem depende da experiência do médico responsável ${ }^{8,9}$ e pode perder sua utilidade quando se trata de um médico não-especialista em IC. Nossos resultados corroboram a falta de acurácia do exame físico cardiovascular, ${ }^{9}$ visto que $11 \%$ dos pacientes foram classificados como de perfil hemodinâmico A e ainda assim considerados casos de IC aguda descompensada. 


\begin{tabular}{|c|c|c|c|c|}
\hline Variáveis & $\begin{array}{c}\text { Total } \\
(\mathrm{N}=463)\end{array}$ & $\begin{array}{c}\text { Pacientes que } \\
\text { receberam alta } \\
\text { hospitalar }(n=421)\end{array}$ & $\begin{array}{l}\text { Pacientes falecidos } \\
\quad(n=42)\end{array}$ & Valor-p \\
\hline \multicolumn{5}{|l|}{ Demográficas } \\
\hline Idade, anos $\pm \mathrm{DP}$ & $61 \pm 16$ & $61 \pm 15$ & $58 \pm 17$ & 0,27 \\
\hline Sexo masculino, $n(\%)$ & $141(30)$ & $127(30)$ & $14(33)$ & 0,67 \\
\hline \multicolumn{5}{|l|}{ Etiologia da insuficiência cardíaca } \\
\hline Isquêmica, $\mathrm{n}(\%)$ & $155(33)$ & $141(33)$ & $14(33)$ & 0,98 \\
\hline Doença de Chagas, $n(\%)$ & $53(11)$ & $43(10)$ & $10(24)$ & 0,008 \\
\hline \multicolumn{5}{|l|}{ Comorbidades } \\
\hline Hipertensão, n (\%) & $318(69)$ & $290(69)$ & $28(67)$ & 0,77 \\
\hline Fibrilação atrial, $\mathrm{n}(\%)$ & $109(23)$ & $101(23)$ & $8(19)$ & 0,51 \\
\hline Diabetes mellitus, $n(\%)$ & $177(38)$ & $164(39)$ & $13(31)$ & 0,31 \\
\hline Insuficiência renal crônica, n (\%) & $98(21)$ & $81(19)$ & $17(40)$ & 0,001 \\
\hline Dislipidemia, n (\%) & $162(35)$ & $150(36)$ & $12(29)$ & 0,36 \\
\hline Depressão, n (\%) & $52(11)$ & $50(12)$ & $2(5)$ & 0,16 \\
\hline Histórico de acidente vascular cerebral, n (\%) & $56(12)$ & $46(11)$ & $10(24)$ & 0,015 \\
\hline Histórico de câncer, $n(\%)$ & $18(4)$ & $14(3)$ & $4(9)$ & 0,048 \\
\hline \multicolumn{5}{|l|}{ Tratamento } \\
\hline Betabloqueador, n (\%) & $273(66)$ & $241(64)$ & $32(82)$ & 0,023 \\
\hline Inibidor de ECA/BRA, n (\%) & $274(59)$ & $251(60)$ & $23(55)$ & 0,50 \\
\hline Diuréticos tiazídicos e de alça, $\mathrm{n}(\%)$ & $311(67)$ & $277(66)$ & $34(81)$ & 0,046 \\
\hline Bloqueadores de canais de cálcio, $\mathrm{n}(\%)$ & $28(7)$ & $25(7)$ & $3(8)$ & 0,80 \\
\hline Digitálicos, $\mathrm{n}(\%)$ & $121(29)$ & $102(27)$ & $19(50)$ & 0,005 \\
\hline Espironolactona, $\mathrm{n}(\%)$ & $182(44)$ & $156(41)$ & $26(67)$ & 0,002 \\
\hline Estatinas, $n(\%)$ & $139(33)$ & $127(34)$ & $12(31)$ & 0,71 \\
\hline \multicolumn{5}{|l|}{ Hemodinâmica } \\
\hline Frequência cardiaca, bpm $\pm \mathrm{DP}$ & $90 \pm 23$ & $90 \pm 23$ & $82 \pm 21$ & 0,025 \\
\hline Pressão arterial sistólica, $\mathrm{mmHg} \pm \mathrm{DP}$ & $121 \pm 29$ & $122 \pm 30$ & $112 \pm 26$ & 0,036 \\
\hline Pressão arterial diastólica, $\mathrm{mmHg} \pm \mathrm{DP}$ & $76 \pm 19$ & $77 \pm 19$ & $70 \pm 14$ & 0,020 \\
\hline Pressão de pulso, $\mathrm{mmHg} \pm \mathrm{DP}$ & $45 \pm 18$ & $45 \pm 18$ & $43 \pm 18$ & 0,30 \\
\hline Pressão de pulso proporcional, $\% \pm$ DP & $37 \pm 9$ & $37 \pm 9$ & $37 \pm 8$ & 0,75 \\
\hline $\mathrm{IHA}, \mathrm{mmHg} \cdot \mathrm{bpm} \pm \mathrm{DP}$ & $4 \pm 2$ & $4 \pm 2$ & $3 \pm 2$ & 0,08 \\
\hline $\mathrm{HA}<4$ mmHg.bpm, $\mathrm{n}(\%)$ & $273(60)$ & $240(57)$ & $33(79)$ & 0,007 \\
\hline FEVE, $\% \pm \mathrm{DP}$ & $27 \pm 8$ & $27 \pm 8$ & $25 \pm 6$ & 0,20 \\
\hline \multicolumn{5}{|l|}{ Perfil hemodinâmico } \\
\hline$A, \%$ & $49(11)$ & $45(11)$ & $4(10)$ & 0,81 \\
\hline B, \% & $311(67)$ & $288(68)$ & $23(55)$ & 0,07 \\
\hline$C, \%$ & $81(17)$ & $68(16)$ & $13(30)$ & 0,02 \\
\hline$L, \%$ & $22(5)$ & $20(5)$ & $2(5)$ & 0,99 \\
\hline \multicolumn{5}{|l|}{ Exames laboratoriais* } \\
\hline Hematócrito, $\% \pm \mathrm{DP}$ & $40 \pm 7$ & $40 \pm 6$ & $38 \pm 9$ & 0,07 \\
\hline Hemoglobina, g/dL $\pm \mathrm{DP}$ & $13 \pm 2$ & $13 \pm 2$ & $13 \pm 2$ & 0,26 \\
\hline Creatinina, $\mathrm{mg} / \mathrm{dL} \pm \mathrm{DP}$ & $1,5 \pm 0,9$ & $1,5 \pm 0,8$ & $1,9 \pm 0,9$ & 0,001 \\
\hline Ureia, $\mathrm{mg} / \mathrm{dL} \pm \mathrm{DP}$ & $68 \pm 41$ & $65 \pm 38$ & $100 \pm 50$ & $<0,001$ \\
\hline Sódio, $m E q / L \pm D P$ & $137 \pm 13$ & $138 \pm 14$ & $136 \pm 6$ & 0,51 \\
\hline
\end{tabular}

DP: desvio padrão; ECA: enzima conversora da angiotensina; BRA: bloqueadores dos receptores da angiotensina; IHA: índice hemodinâmico agudo; FEVE: fração de ejeção do ventrículo esquerdo. Valor-p calculado a partir da comparação univariada entre ambos os grupos. ${ }^{*} N=412$. 
Tabela 2 - Sensibilidade, especificidade, AUC com IC de 95\% e melhores valores de corte para mortalidade intra-hospitalar em pacientes com insuficiência cardíaca aguda descompensada com fração de ejeção reduzida

\begin{tabular}{|c|c|c|c|c|c|}
\hline \multirow{2}{*}{$\begin{array}{l}\text { Parâmetros prognósticos } \\
\text { propostos }\end{array}$} & \multicolumn{4}{|c|}{ Análise univariada } & \multirow{2}{*}{$\begin{array}{c}\text { Comparação com AUC } \\
\text { considerando valor de } \\
\text { IHA } \leq 4 \mathrm{mmHg} \cdot \mathrm{bpm}\end{array}$} \\
\hline & Sensibilidade & Especificidade & AUC (IC 95\%) & Valor-p & \\
\hline $\mathrm{IHA} \leq 4 \mathrm{mmHg} \cdot \mathrm{bpm}$ & 0,786 & 0,429 & $0,607(0,540-0,674)$ & 0,01 & --- \\
\hline \multicolumn{6}{|l|}{ Frequência cardíaca } \\
\hline$\leq 74 \mathrm{bpm}$ & 0,309 & 0,750 & $0,530(0,456-0,604)$ & 0,39 & --- \\
\hline$\leq 88 \mathrm{bpm}$ & 0,667 & 0,513 & $0,590(0,514-0,666)$ & 0,03 & 0,048 \\
\hline$\leq 104 \mathrm{bpm}$ & 0,857 & 0,254 & $0,556(0,498-0,613)$ & 0,58 & --- \\
\hline \multicolumn{6}{|l|}{ Pressão arterial sistólica } \\
\hline$\leq 100$ & 0,452 & 0,698 & $0,575(0,496-0,654)$ & 0,04 & 0,450 \\
\hline$\leq 120$ & 0,738 & 0,430 & $0,584(0,513-0,655)$ & 0,04 & 0,570 \\
\hline$\leq 140$ & 0,905 & 0,190 & $0,547(0,498-0,596)$ & 0,14 & --- \\
\hline \multicolumn{6}{|l|}{ Pressão arterial diastólica } \\
\hline$\leq 60$ & 0,453 & 0,741 & $0,596(0,518-0,676)$ & 0,01 & 0,830 \\
\hline$\leq 73$ & 0,643 & 0,513 & $0,578(0,500-0,655)$ & 0,06 & --- \\
\hline$\leq 84$ & 0,857 & 0,257 & $0,557(0,499-0,614)$ & 0,11 &.--- \\
\hline
\end{tabular}

AUC: área sob a curva ROC (Receiving Operating Characteristic); IC: intervalo de confiança; IHA: índice hemodinâmico agudo.

A medição da frequência cardíaca e a pressão arterial está disponível em praticamente qualquer estabelecimento de saúde, tem boa acurácia e requer treinamento mínimo. ${ }^{11,12}$ Estudos anteriores tentaram utilizar estes parâmetros como fatores prognósticos na IC aguda descompensada. A relação entre frequência cardíaca e prognóstico é conhecida há décadas, e com o advento dos betabloqueadores e, mais recentemente, da ivabradina, frequências cardíacas baixas são um alvo no tratamento da IC estável. ${ }^{19}$ Além disso, a incompetência cronotrópica também é um marcador de risco: pacientes cujas frequências cardíacas não aumentam durante o exercício físico têm prognósticos piores que aqueles com frequências cardíacas de reserva normais, mesmo com o uso de betabloqueadores. ${ }^{20,21}$ Apesar de estudos terem determinado o aumento esperado na frequência cardíaca durante um teste de esforço, ${ }^{20,21}$ não há valores de referência para o aumento da frequência cardíaca em situações do tipo "luta ou fuga" como é o caso das admissões em serviços de emergência. Pacientes japoneses com IC aguda descompensada admitidos com frequências cardíacas acima de 120 bpm apresentaram menores índices de mortalidade que aqueles admitidos com frequências cardíacas menores. ${ }^{15}$ Entretanto, uma alta frequência cardíaca foi considerada um preditor independente de mortalidade a curto prazo em pacientes com IC aguda descompensada em outros estudos. ${ }^{16,30,31}$

O registro OPTIMIZE-HF14 observou que pressão arterial sistólica abaixo de $120 \mathrm{mmHg}$ identificava pacientes com IC aguda descompensada que possuíam um prognóstico ruim apesar dos tratamentos. Baixa pressão arterial sistólica também indicou risco a curto prazo numa coorte europeia. ${ }^{13}$ Neste estudo, a pressão arterial abaixo de $120 \mathrm{mmHg}$ não estava independentemente relacionada à mortalidade de acordo com a análise multivariada. Pacientes do registro BREATHE eram mais jovens, e os protocolos de tratamento estavam mais atualizados que aqueles utilizados nos outros estudos (quase uma década antes). Além disso, esses dois estudos ${ }^{13,14}$ incluíram pacientes com fração de ejeção preservada ou reduzida, e o valor prognóstico da pressão arterial pode variar de acordo com a fração de ejeção do ventrículo esquerdo. ${ }^{32}$ Baixa pressão de pulso foi definida como um preditor independente de mortalidade na IC aguda descompensada pelo estudo VMAC-HF. ${ }^{33} \mathrm{O}$ tratamento da IC evoluiu substancialmente desde então, o que pode explicar a falta de poder prognóstico da pressão de pulso em nosso estudo.

A interação intrínseca entre pressão arterial e frequência cardíaca e como elas são influenciadas por medicamentos para IC pode ter influenciado os resultados de estudos prévios considerando cada um destes parâmetros. Até onde sabemos, este é o primeiro estudo a apresentar um índice que combina a análise da frequência cardíaca e pressão de pulso de pacientes com IC aguda descompensada e demonstrar que sua capacidade como ferramenta prognóstica é superior àquelas da frequência cardíaca ou pressão arterial sozinhas.

\section{Limitações}

Esta análise possui limitações. Primeiramente, a mortalidade intra-hospitalar foi baseada no registro feito por pesquisadores e não foi confirmada. Na realidade, registros são estudos observacionais, e análises quanto à adequação do tratamento a cada paciente não estavam no escopo de nosso estudo. Considerando que nosso principal objetivo foi analisar a utilidade de um índice facilmente obtido no momento da 


\begin{tabular}{|c|c|c|c|c|c|c|c|c|c|c|}
\hline & \multicolumn{2}{|c|}{ Modelo 1} & \multicolumn{2}{|c|}{ Modelo 2} & \multicolumn{2}{|c|}{ Modelo 3} & \multicolumn{2}{|c|}{ Modelo 4} & \multicolumn{2}{|c|}{ Modelo 5} \\
\hline AIC & \multicolumn{2}{|l|}{137,0} & \multicolumn{2}{|l|}{136,3} & \multicolumn{2}{|l|}{135,6} & \multicolumn{2}{|l|}{135,7} & \multicolumn{2}{|l|}{133,7} \\
\hline $\begin{array}{l}\text { Valor-p } \\
\text { em comparação com Modelo } 0\end{array}$ & \multicolumn{2}{|l|}{0,294} & \multicolumn{2}{|l|}{0,183} & \multicolumn{2}{|l|}{0,113} & \multicolumn{2}{|l|}{0,116} & \multicolumn{2}{|l|}{0,035} \\
\hline Parâmetro & $\begin{array}{l}\mathrm{RC} \\
\mathrm{IC} 95 \%\end{array}$ & $p$ & $\begin{array}{c}\mathrm{RC} \\
\text { IC } 95 \%\end{array}$ & $\mathrm{p}$ & $\begin{array}{c}\mathrm{RC} \\
\mathrm{IC} 95 \%\end{array}$ & $\mathrm{p}$ & $\begin{array}{l}\mathrm{RC} \\
\text { IC } 95 \%\end{array}$ & $\mathrm{p}$ & $\begin{array}{l}\mathrm{RC} \\
\mathrm{IC} 95 \%\end{array}$ & $p$ \\
\hline Doença de Chagas & $\begin{array}{c}0,089 \\
0,006-0,171\end{array}$ & 0,035 & $\begin{array}{c}0,784 \\
-0,006-0,163\end{array}$ & 0,071 & $\begin{array}{c}0,080 \\
-0,003-0,164\end{array}$ & 0,060 & $\begin{array}{c}0,777 \\
-0,006-0,162\end{array}$ & 0,071 & $\begin{array}{c}0,765 \\
-0,007-0,160\end{array}$ & 0,072 \\
\hline DRC & $\begin{array}{c}0,104 \\
0,041-0,167\end{array}$ & 0,001 & $\begin{array}{c}0,104 \\
0,040-0,167\end{array}$ & 0,001 & $\begin{array}{c}0,107 \\
0,044-0,170\end{array}$ & 0,001 & $\begin{array}{c}0,100 \\
0,037-0,164\end{array}$ & 0,002 & $\begin{array}{c}0,112 \\
0,048-0,175\end{array}$ & 0,001 \\
\hline $\begin{array}{l}\text { Histórico de acidente vascular } \\
\text { cerebral }\end{array}$ & $\begin{array}{c}0,840 \\
0,054-0,163\end{array}$ & 0,036 & $\begin{array}{c}0,089 \\
0,011-0,168\end{array}$ & 0,025 & $\begin{array}{c}0,093 \\
0,014-0,170\end{array}$ & 0,021 & $\begin{array}{c}0,858 \\
0,007-0,164\end{array}$ & 0,032 & $\begin{array}{c}0,092 \\
0,013-0,169\end{array}$ & 0,022 \\
\hline Histórico de câncer & $\begin{array}{c}0,143 \\
0,011-0,276\end{array}$ & 0,033 & $\begin{array}{c}0,148 \\
0,016-0,281\end{array}$ & 0,028 & $\begin{array}{c}0,139 \\
0,007-0,271\end{array}$ & 0,039 & $\begin{array}{c}0,139 \\
0,007-0,272\end{array}$ & 0,038 & $\begin{array}{c}0,140 \\
0,009-0,273\end{array}$ & 0,037 \\
\hline Betabloqueadores & $\begin{array}{c}0,168 \\
-0,40-0,073\end{array}$ & 0,563 & $\begin{array}{c}0,196 \\
-0,037-0,076\end{array}$ & 0,497 & $\begin{array}{c}0,180 \\
-0,038-0,074\end{array}$ & 0,531 & $\begin{array}{c}0,021 \\
-0,035-0,077\end{array}$ & 0,463 & $\begin{array}{c}0,172 \\
-0,394-0,073\end{array}$ & 0,551 \\
\hline Diuréticos tiazídicos e de alça & $\begin{array}{c}-0,005 \\
-0,066-0,057\end{array}$ & 0,887 & $\begin{array}{c}-0,003 \\
-0,065-0,058\end{array}$ & 0,918 & $\begin{array}{c}-0,005 \\
-0,066-0,057\end{array}$ & 0,884 & $\begin{array}{c}-0,004 \\
-0,065-0,058\end{array}$ & 0,909 & $\begin{array}{c}-0,006 \\
-0,068-0,056\end{array}$ & 0,850 \\
\hline Digitálicos & $\begin{array}{c}0,053 \\
-0,009-0,115\end{array}$ & 0,096 & $\begin{array}{c}0,056 \\
-0,005-0,117\end{array}$ & 0,073 & $\begin{array}{c}0,538 \\
-0,007-0,115\end{array}$ & 0,086 & $\begin{array}{c}-0,003 \\
-0,007-0,115\end{array}$ & 0,086 & $\begin{array}{c}0,515 \\
-0,009-0,113\end{array}$ & 0,100 \\
\hline Espironolactona & $\begin{array}{c}0,540 \\
-0,004-0,112\end{array}$ & 0,068 & $\begin{array}{c}0,527 \\
-0,005-0,110\end{array}$ & 0,075 & $\begin{array}{c}0,053 \\
-0,004-0,111\end{array}$ & 0,071 & $\begin{array}{c}0,053 \\
-0,004-0,111\end{array}$ & 0,072 & $\begin{array}{c}0,053 \\
-0,005-0,110\end{array}$ & 0,074 \\
\hline Frequência cardíaca $\leq 88 \mathrm{bpm}$ & $\begin{array}{c}0,277 \\
-0,025-0,080\end{array}$ & 0,627 & & & & & & & & \\
\hline $\begin{array}{l}\text { Pressão arterial sistólica } \\
\leq 100 \mathrm{mmHg}\end{array}$ & & & $\begin{array}{c}0,380 \\
-0,018-0,094\end{array}$ & 0,188 & & & & & & \\
\hline$\leq 120 \mathrm{mmHg}$ & & & & & $\begin{array}{c}0,042 \\
-0,010-0,095\end{array}$ & 0,117 & & & & \\
\hline $\begin{array}{l}\text { Pressão arterial diastólica } \leq 60 \\
\mathrm{mmHg}\end{array}$ & & & & & & & $\begin{array}{c}0,046 \\
-0,012-0,105\end{array}$ & 0,121 & & \\
\hline $\mathrm{IHA} \leq 4 \mathrm{mmHglbpm}$ & & & & & & & & & $\begin{array}{c}0,061 \\
0,007-0,114\end{array}$ & 0,025 \\
\hline
\end{tabular}

AIC: critério de informação de Akaike; RC: razão de chance; IC: intervalo de confiança; DRC: doença renal crônica; IHA: índice hemodinâmico agudo. 0 Modelo 0 incluiu doença de Chagas como etiologia da insuficiência cardíaca, doença renal crônica, histórico de câncer, uso contínuo de betabloqueadores, diuréticos tiazídicos e de alça, digitálicos e espironolactona. Os Modelos 1 a 5 incluíram todas as variáveis do Modelo 0 mais um parâmetro e valor de corte da seguinte forma: o Modelo 1 considerou frequência cardíaca $\leq 88 \mathrm{bpm}$; o Modelo 2 considerou pressão arterial sistólica $\leq 100 \mathrm{mmHg}$, o Modelo 3 considerou pressão arterial sistólica $\leq 120 \mathrm{mmHg}$, o Modelo 4 considerou pressão arterial diastólica $\leq 60 \mathrm{mmHg}$ e o Modelo 5 considerou IHA $\leq 4 \mathrm{mmHglbpm}$.

admissão do paciente no setor de emergência e que os testes de troponina e BNP (peptídeo natriurético cerebral) não estão disponíveis em todos os estabelecimentos de saúde brasileiros, parâmetros laboratoriais não foram incluídos em nosso modelo.

Os dados do registro não foram obtidos através de um protocolo padronizado, portanto as medições de pressão arterial e frequência cardíaca podem ter sido realizadas com equipamentos diferentes. De qualquer maneira, estes são sinais vitais que requerem treinamento mínimo para sua medição. ${ }^{11,12}$ Além disso, o fato de não existir uma padronização neste caso aumenta a aplicabilidade clínica do nosso índice, já que reflete resultados reais.
É importante notar que os resultados apresentados neste estudo se restringem a pacientes com fração de ejeção reduzida. O estudo foi conduzido entre 2011 e 2012, antes da aprovação de medicamentos novos como a ivabradina e sacubitril-valsartana, ${ }^{19}$ que poderiam influenciar O IHA.

A população brasileira é muito diversa quanto à etnia e acesso a estabelecimentos de saúde, e este estudo incluiu hospitais públicos e privados de todas as regiões do país. ${ }^{23}$ Embora a generalização de nossos resultados possa ser limitada, é importante enfatizar que os dados clínicos e demográficos dos pacientes incluídos neste estudo são semelhantes aos de outras coortes. ${ }^{14,16,30,31}$ 


\section{Artigo Original}

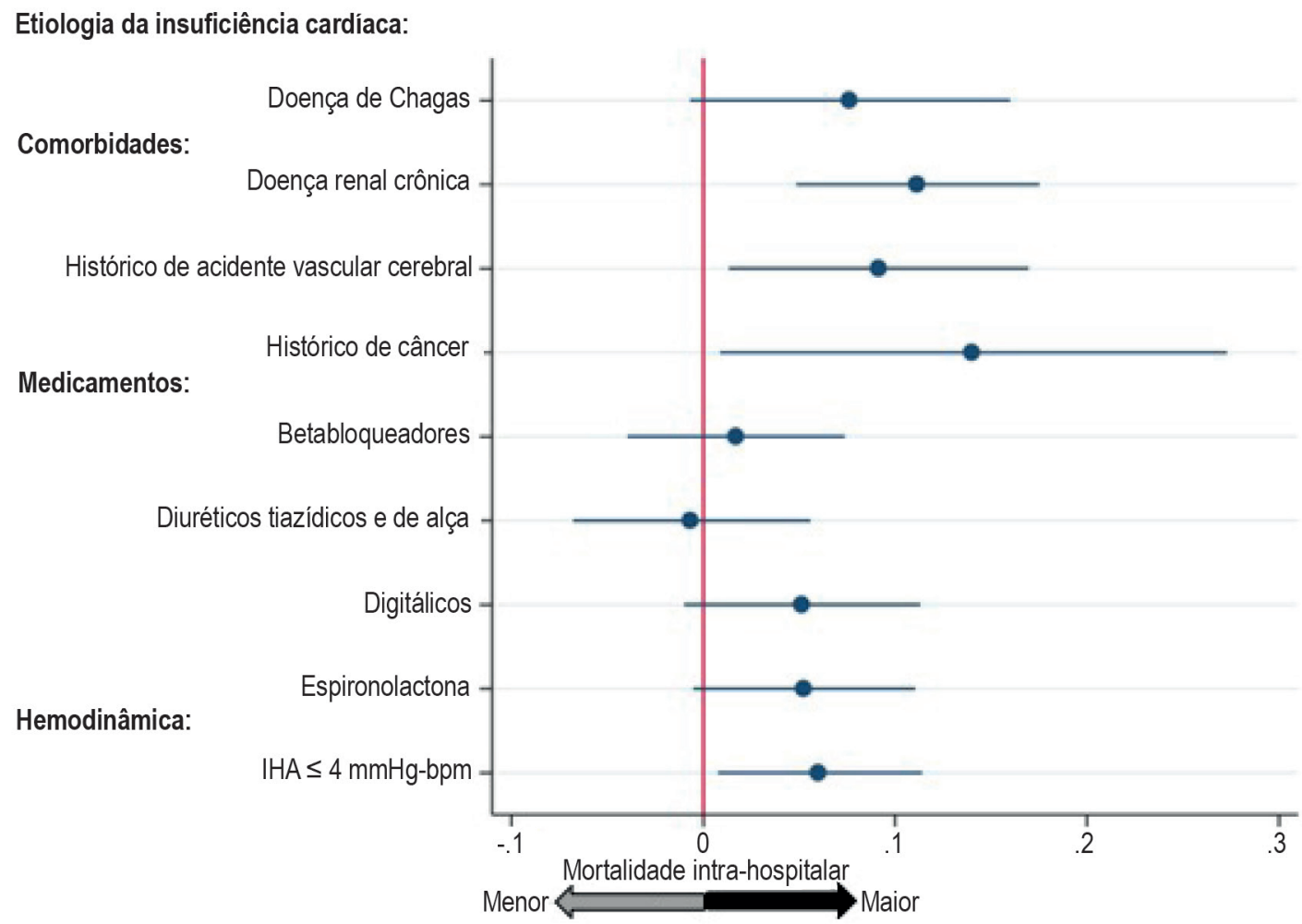

Figura 2 - Razão de chance, calculada a partir de um modelo de regressão multivariada que incluiu etiologia da insuficiência cardíaca, comorbidades, uso de medicamentos e índice hemodinâmico de pacientes admitidos com insuficiência cardíaca aguda descompensada com fração de ejeção reduzida ( $N=463)$. IHA: índice hemodinâmico agudo.

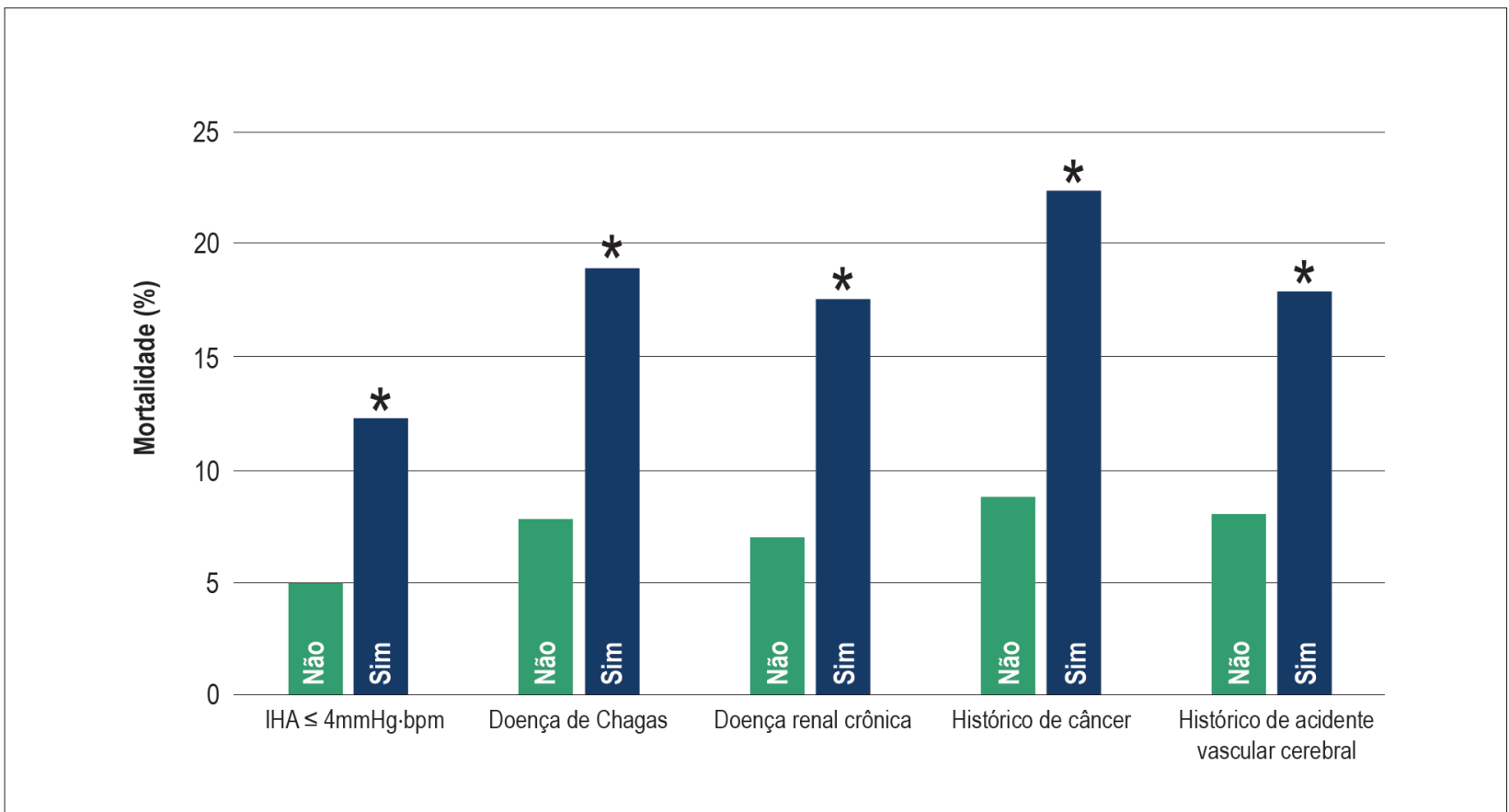

Figura 3 - Índices de mortalidade intra-hospitalar em pacientes com insuficiência cardíaca aguda descompensada com fração de ejeção reduzida de acordo com a presença de fatores prognósticos. * $p<0,05$ quando comparado ao "Não" no mesmo índice prognóstico. 
É importante ressaltar que a AUC da análise do IHA foi relativamente pequena. Mesmo assim, sua sensibilidade foi boa e pode ser útil na orientação de médicos emergencistas na triagem de pacientes.

\section{Conclusão}

Diferentes fatores prognósticos têm sido propostos para a IC aguda descompensada. Entretanto, muitos dependem de biomarcadores, treinamento de pessoal e recursos tecnológicos, que nem sempre estão disponíveis. O IHA consiste num fator prognóstico de mortalidade intra-hospitalar prático, objetivo e facilmente obtido em pacientes com IC aguda descompensada. Futuros estudos prospectivos deverão avaliar a reprodutibilidade destes resultados em outras populações.

\section{Agradecimentos}

Os autores agradecem aos investigadores do registro BREATHE: Helder José Lima Reis, Paulo Roberto Nogueira, Ricardo Pavanello, Luiz Claudio Danzmann, Elizabete Silva dos Santos, Mucio Tavares de Oliveira Filho, Silvia Marinho Martins, Marcelo lorio Garcia, Antonio Baruzzi, Maria Alayde Mendonça da Silva, Ricardo Gusmão, Aguinaldo Figueiredo de Freitas Júnior, Fernando Carvalho Neuenschwander, Manoel Fernandes Canesin, Eduardo Darzé, Mauro Esteves Hernandes, Ricardo Mourilhe Rocha, Antonio Carlos Sobral Sousa, Jose Albuquerque de Figueiredo Neto, Renato D. Lopes, Jacqueline Sampaio, Estêvão Lanna Figueiredo, Abilio Augusto Fragata Filho, Alvaro Rabelo Alves Júnior, Carlos V. Nascimento, Antonio Carlos Pereira-Barretto, Fabio Serra Silveira, Gilson Soares Feitosa, Conrado Roberto Hoffmann Filho, Humberto Villacorta Júnior, Sidney Araújo, Beatriz

\section{Referências}

1. Ambrosy AP, Fonarow GC, Butler J, Chioncel O, Greene SJ, Vaduganathan $\mathrm{M}$, et al. The global health and economic burden of hospitalizations for heart failure: lessons learned from hospitalized heart failure registries. J Am Coll Cardiol. 2014;63(12):1123-33.

2. Nicol ED, Fittall B, Roughton M, Cleland JG, Dargie H, Cowie MR. NHS heart failure survey: a survey of acute heart failure admissions in England, Wales and Northern Ireland. Heart. 2008;94(2):172-7.

3. Long B, Koyfman A, Gottlieb M. Management of heart failure in the emergency department setting: an evidence-based review of the literature. J Emerg Med. 2018;55(5):635-46.

4. Nohria A, Mielniczuk LM, Stevenson LW. Evaluation and monitoring of patients with acute heart failure syndromes. Am J Cardiol. 2005;96(6 suppl):32-40.

5. Thibodeau JT, Drazner MH. The Role of the clinical examination in patients with heart failure. JACC Heart Fail. 2018;6(7):543-51.

6. Nohria A, Tsang SW, Fang JC, Lewis EF, Jarcho JA, Mudge GH, et al. Clinical assessment identifies hemodynamic profiles that predict outcomes in patients admitted with heart failure. J Am Coll Cardiol. 2003;41(10):1797-804.

7. Drazner MH, Hellkamp AS, Leier CV, Shah MR, Miller LW, Russell SD, et al. Value of clinician assessment of hemodynamics in advanced heart failure: the ESCAPE trial. Circ Heart Fail. 2008;1(3):170-7.
Bojikian Matsubara, Otávio Gebara, Gustavo Luiz Gouvea de Almeida, Maria da Consolação Vieira Moreira, Roberto Luiz Marino, João Miguel de Malta Dantas, Marcelo Imbroinise Bittencourt, Marcelo Silveira Teixeira, Elias Pimentel Gouvea, Marcus Vinícius Simões, Renato Jorge Alves, Fabio Villas-Boas, Charles Mady, Felipe Montes Pena, Eduardo Costa, Sabrina Bernardez-Pereira, Otavio Berwanger.

\section{Contribuição dos autores}

Concepção e desenho da pesquisa: Castro RRT, Albuquerque DC, Rohde LE, Almeida D, David J, Rassi S, Bacal F, Bocchi E, Moura L; Obtenção de dados: Lechnewski L, Homero A, Albuquerque DC, Rohde LE, Almeida D, David J, Rassi S, Bacal F, Bocchi E, Moura L; Análise e interpretação dos dados e Análise estatística: Castro RRT; Redação do manuscrito: Castro RRT, Lechnewski L; Revisão crítica do manuscrito quanto ao conteúdo intelectual importante: Albuquerque DC, Rohde LE, Almeida D, David J, Rassi S, Bacal F, Bocchi E, Moura L.

\section{Potencial conflito de interesses}

Os autores declaram não haver conflito de interesses pertinentes.

\section{Fontes de financiamento}

O presente estudo não contou com fontes de financiamento externas.

\section{Vinculação acadêmica}

Não há vinculação deste estudo a programas de pósgraduação.

8. Frea S, Pidello S, Canavosio FG, Bovolo V, Botta M, Bergerone S, et al. Clinical assessment of hypoperfusion in acute heart failure - evergreen or antique? Circ J. 2015;79(2):398-405

9. Stevenson LW, Perloff JK. The limited reliability of physical signs for estimating hemodynamics in chronic heart failure. JAMA. 1989;261(6):884-8.

10. Castro RRT, Joyce E, Lakdawala NK, Stewart G, Nohria A, Givertz MM, et al Patients report more severe daily limitations than recognized by their physicians. Clin Cardiol. 2019;42(12):1181-8.

11. Chen Z, Wang X, Wang Z, Zhang L, Hao G, Dong Y, et al. Assessing the validity of oscillometric device for blood pressure measurement in a large populationbased epidemiologic study. J Am Soc Hypertens. 2017;11(11):730-6.

12. OpioMO, KellettJ, Kitovu Hospital Study Group. How well are pulses measured? practice-based evidence from an observational study of acutely III medical patients during hospital admission. Am J Med. 2017;130(7):863.e13-16.

13. Harjola VP, Follath F, Nieminen MS, Brutsaert D, Dickstein K, Drexler H, et al Characteristics, outcomes, and predictors of mortality at 3 months and 1 year in patients hospitalized for acute heart failure. Eur J Heart Fail. 2010;12(3):239-48.

14. Gheorghiade M, Abraham WT, Albert NM, Greenberg BH, O'Connor CM, She $\mathrm{L}$, et al. Systolic blood pressure at admission, clinical characteristics, and outcomes in patients hospitalized with acute heart failure. JAMA. 2006;296(18):2217-26. 
15. Kajimoto K, Sato N, Keida T, Sakata Y, Asai K, Mizuno M, et al. Low admission heart rate is a marker rather than a mediator of increased in-hospital mortality for patients with acute heart failure syndromes in sinus rhythm. Int J Cardiol. 2014;171(1):98-100.

16. Kaplon-Cieslicka A, Balsam P, Ozieranski K, Tymińska A, Peller M, Galas M, et al. Resting heart rate at hospital admission and its relation to hospital outcome in patients with heart failure. Cardiol J. 2014;21(4):425-33.

17. Lancellotti P, Ancion A, Magne J, Ferro G, Pierard LA. Elevated heart rate at 24$36 \mathrm{~h}$ after admission and in-hospital mortality in acute in non-arrhythmic heart failure. Int J Cardiol. 2015 Mar 1;182:426-30.

18. Ibrahim NE, Januzzi JL, Rabideau DJ, Gandhi PU, Gaggin HK. Serial heart rates, guideline-directed beta blocker use, and outcomes in patients with chronic heart failure with reduced ejection fraction. Am J Cardiol. 2017;120(5):803-8.

19. Yancy CW, Jessup M, Bozkurt B, Butler J, Casey Jr DE, Colvin MM, et al. 2016 ACC/AHA/HFSA focused update on new pharmacological therapy for heart failure: an update of the $2013 \mathrm{ACCF} / \mathrm{AHA}$ guideline for the management of heart failure: a report of the American College of Cardiology/American Heart Association task force on clinical practice guidelines and the Heart Failure Society of America. Circulation. 2016;134(13):e282-93.

20. Benes J, Kotrc M, Borlaug BA, Lefflerova K, Jarolim P, Bendlova B, et al. Resting heart rate and heart rate reserve in advanced heart failure have distinct pathophysiologic correlates and prognostic impact: a prospective pilot study. JACC Heart Fail. 2013;1(3):259-66.

21. Dobre D, Zannad F, Keteyian SJ, Stevens SR, Rossignol P, Kitzman DW, et al. Association between resting heart rate, chronotropic index, and long-term outcomes in patients with heart failure receiving beta-blocker therapy: data from the HF-ACTION trial. Eur HeartJ. 2013;34(29):2271-80.

22. LinckC, Phillips S. Fight or flight? Disruptive behavior in medical/surgical services. Nurs Manage. 2005;36(5):47-51.

23. Albuquerque DC, Souza Neto JD, Bacal F, Rohde LEP, Bernadez-Pereira S, Berwanger O, et al. I Brazilian registry of heart failure - clinical aspects, care quality and hospitalization outcomes. Arq Bras Cardiol. 2015;104(6):433-42.
24. BREATHE. Rationale and design: BREATHE registry--I Brazilian registry of heart failure. Arq Bras Cardiol. 2013;100(5):390-4.

25. Marantz PR, Tobin JN, Wassertheil-Smoller S, Steingart RM, Wexler JP, Budner N, et al. The relationship between left ventricular systolic function and congestive heart failure diagnosed by clinical criteria. Circulation. 1988;77(3):607-12.

26. Akaike $\mathrm{H}$. A new look at the statistical model identification. IEEE Trans Automat Contr. 1974;19(6):716-23.

27. Fonseca C, Araujo I, Marques F, Bras D, Bettencourt P. A closer look at acute heart failure: putting Portuguese and European data into perspective. Rev Port Cardiol. 2016;35(5):291-304.

28. Cohen-Solal A, Laribi S, Ishihara S, Vergaro G, Baudet M, Logeart D, et al. Prognostic markers of acute decompensated heart failure: the emerging roles of cardiac biomarkers and prognostic scores. Arch Cardiovasc Dis. 2015;108(1):64-74.

29. Negi S, Sawano M, Kohsaka S, Inohara T, Shiraichi Y, Kohno T, et al. Prognostic implication of physical signs of congestion in acute heart failure patients and its association with steady-state biomarker levels. PLoS One. 2014;9(5):e96325.

30. Lee DS, Stitt A, Austin PC, Stukel TA, Schull MJ, Chong A, et al. Prediction of heart failure mortality in emergent care: a cohort study. Ann Intern Med. 2012;156(11):767-75

31. O'Connor CM, Mentz RJ, Cotter G, Metra M, Cleland JG, Davison BA, et al. The PROTECT in-hospital risk model: 7-day outcome in patients hospitalized with acute heart failure and renal dysfunction. Eur J Heart Fail. 2012;14(6):605-12.

32. Jackson CE, Castagno D, Maggioni AP, Køber L, Squire IB, Swedberg K, et al. Differing prognostic value of pulse pressure in patients with heart failure with reduced or preserved ejection fraction: results from the MAGGIC individual patient meta-analysis. Eur Heart J. 2015;36(18):1106-14.

33. Aronson D, Burger AJ. Relation between pulse pressure and survival in patients with decompensated heart failure. Am J Cardiol. 2004;93(6):785-8. 